\title{
Line Olsen-Ring/Gerhard Ring Die registrierte (Lebens-) Partnerschaft im Recht der skandinavischen Staaten
}

Homosexuelle Partnerschaften sollen in Zukunft in Deutschland rechtlich besser gestellt werden. Bislang gibt es bei solchen Partnerschaften keine Unterbaltsansprüche und kein Erbrecht. Die Autoren skizzieren die gegenüber Deutschland fortschrittlichere gesetzliche Lage in den skandinavischen Ländern. Sie wollen damit Anregungen für die in der Bundesrepublik anstehende rechtspolitische Diskussion geben. Eine "registrierte Partnerschaft" hat im Recht der skandinavischen Länder die Begründung gegenseitiger gesetzlicher Unterhaltsansprüche und das Erbrecht zur Folge.

Die Red.

Nach dem Wahlsieg von SPD und Bündnis 9o/Die Grünen bei der Bundestagswahl vom 27. September 1998 hat sich die rot-grüne Regierungskoalition in der Justizpolitik u.a. darauf verständigt, die Minderheitenrechte zu stärken. Unter diesem Gesichtspunkt bestimmt Kapitel IX. der Koalitionsvereinbarung vom 20. Oktober I998 unter Ziffer 10. (Minderheitenrechte), daß die neue Bundesregierung Minderheiten schützen und ihre Gleichberechtigung und gesellschaftliche Teilhabe erreichen will: "Niemand darf wegen seiner Behinderung, Herkunft, Hautfarbe, ethnischer Zugehörigkeit oder sexueller Orientierung als Schwuler oder Lesbe diskriminiert werden. Dazu werden wir ein Gesetz gegen Diskriminierung und zur Förderung der Gleichbehandlung (u. a. mit der Einführung des Rechtsinstiturs der eingetragenen Lebenspartnerschaft mit Rechten und Pflichten) auf den Weg bringen. Die Empfehlungen des Europäischen Parlaments zur Gleichberechtigung von Lesben und Schwulen werden berücksichtigt«.

Die neue Justizministerin H. Däubler-Gmelin hat zu diesem Aspekt der Justizpolitik erläuternd festgestellt²: »Für homosexuelle Partnerschaften gibt es heute keinen gesetzlichen Unterhaltsanspruch, kein gesetzliches Erbrecht und auch nicht das Recht, nach dem Tod des Partners die gemeinsame Wohnung zu übernehmen. Diese und auch andere Mängel werden wir beseitigen. Stabile Beziehungen zwischen zwei Personen sind wichtig und müssen gefördert werden. Die sexuelle Orientierung der Bürger geht den Staat nichts an « $^{3}$. Ziel sei die Schaffung einer registrierten Partnerschaft, an die sich Rechte und Pflichten knüpfen - jedoch keine neue Form der Ehe. Eine Adoption von Kindern durch gleichgeschlechtliche Paare geht der Justizministerin »erheblich zu weit «. Diese Frage berühre nicht Partnerschaften und deren Rechte, sondern müsse unter dem Gesichtspunkt des Kindeswohles überprüft werden. Im übrigen dürfe - bei Vorliegen der nötigen Voraussetzungen - auch heute schon eine einzelne Person ein Kind adoptierent.

\footnotetext{
I Im Internct abrufbar unter http://www.spd.de/aktuell/programmatisches/vertrag.htm.

2 „Uns karn auch mal was Neues cinfallen*, DIE ZEIT Nr. 46 v. 5. November 1998, S. 8.

3 Daubler-Gmelin, DIE ZF.IT Nr. 46 v. s. November 1998, S. 8 (9).

4 So bestimmt etwa $\$ 174$ t Abs. 2 S. I BGB, daß wer nicht verheiratet ist, ein Kind nur allein annehnen kann.
} 


\section{Ansätze einer rechtspolitischen Debatte in Deutschland und die sog. "Hamburger Ebe"}

Diese allgemeinen politischen Ankündigungen haben jedoch bis zum Sommer 1999 noch keine greifbaren Konsequenzen im Hinblick auf eine tatsächliche gesetzliche Umsetzung auf Bundesebene nach sich gezogen.

Demhingegen wurde vom Hamburger Senat im April eine Umsetzungsverordnung sowie ein Gesetz für die Eintragung gleichgeschlechtlicher Partnerschaften beschlossen, die Verfahrensvorgaben hinsichtlich einer Registrierung beinhalten, welche von der Presse nunmehr vielfach als "Hamburger Ehe« bezeichnet werden. Dabei handelt es sich lediglich um einen Akt »symbolischer Politik «, da der Senat bei der Verabschiedung klarstellte, daß eine neheähnliche Partnerschaft - anders als eine formale Eintragung auf dem Standesamt - nicht durch Landesrecht, sondern nur bundesgesetzlich geregelt werden « kann'.

Hamburg hat allein eine symbolische Anerkennung gleichgeschlechtlicher Partnerschaften getroffen. Der bisherige rechtliche Status der Partner bzw. Partnerinnen wird durch die Registrierung nicht geändert. Die Eintragung zeitigt keinerlei materiell-rechtliche Wirkungen.

Nachdem also hinsichtlich der Etablierung des Rechtsinstituts einer registrierten Lebenspartnerschaft in Deutschland allein die Frage des "Ob" bislang positiv beschieden wurde, bleibt das "Wie«, d.h. die inhaltliche Ausgestaltung der Partnerschaft, immer noch in der Schwebe. Vor diesem Hintergrund kann es für die kommenden rechtspolitischen Überlegungen und Diskussionen in Deutschland ${ }^{6}$ durchaus von Interesse sein, einmal einen Blick auf die Rechtslage in den skandinavischen Staaten ${ }^{7}$ - Dänemark, Norwegen und Schweden - zu werfen, die bereits seit einigen Jahren das Rechtsinstitut einer »registrierten Partnerschaft « anerkannt haben.

Im Hinblick auf die Etablierung des Rechtsinstituts einer registrierten (Lebens-) Partnerschaft kommt Dänemark insoweit eine Vorreiterrolle zu, als das dänische Parlament (Folketinget) als erstes in der Staatengemeinschaft im Jahre 1989 ein entsprechendes Gesetz (lov om registreret partnerskab - Pal DK) ${ }^{8}$ erlassen hat ${ }^{9}$ (dazu unter II.).

In Norwegen datiert das Gesetz über die registrierte Partnerschaft (lov om registrert partnerskap - Nr. 40) ${ }^{10}$ vom 30. April $1993^{11}$ (unter III.). In Schweden gilt das Lag om registrerat partnerskap ${ }^{12}$, das am I. Januar 1995 in Kraft getreten ist ${ }^{13}$ (IV.2.). Schwe-

5 Presseerklärung des Hamburger Senats vom 6. April 1999.

6 Vgl. generell zum Verhaltnis von the und eheähnlichen Gemeinschaften Pawlowski, Abschied von der "burgerlichen Ehea?, JZ r998, 1032.

7 Dazu Ring/Olsen-Ring, Einfuhrung in das Skandinavische Reche (JuS-Schriftenreihe), München r999, Rn. 412 und 460 .

8 Lov $1989-06-07 \mathrm{nr} 372$

9 Dazu näher Karnovs Lovsamline/Nielsen, is. udg., 1997, 5. 4955f. (künftig Dansk Karnov); Nielsen/ Vorstrup Rasmussen/Taksee-Jensen, Familieretten, 2. udg., 1997, Kapitel 15; Lund-Andersen/Munck/ Nørgaard/Høigaard Pedersen/Vesterdorf, Familieret, 4. udg,, 1996, s. 175 ff.; Svenné Schmidr, International person-, familie- og arverct, 1990, s. 40 ff.; Roshoim, Skarrildhusrapporten, 1989, s. 66 ff.; Elmer/ Lund-Larsen, Familieretlige lovxndringer i 1989 , s. 126 ( 134 ff.).

10 Dazu naher Karnov - Norsk kommentert Lovsamling/Strøm Bull, Lov on registrert partnerskap, 5. 2874 ff. (kunftig Norsk Karnov); Strandbakken, Lov om registrert partnerskap, JV 5/6 1993, 300.

Ir Vgl. zu den Vorarbeiten dieses Gesetzes naher Odelstingsproposisjon (Ot.prp.) nr. 32 (1992-93), (Innstilling til Odelstinget) Innst. O. nr. 70 (1992 93).

12 Zum schwedischen Partnerschaftsgesetz naher Agell, Áktenskap, Samboende, Parcnerskap, 2. udg. 1999, Kapitel 14; Bogdan, Registrerat parncrskap och svensk internationell privaträtc, Svensk Juristtidning (SvJT) 1994, 773; Jansson, Partnerskap, Ny Juridik, 1 : 95, 7; Karnov - Svensk lagsamling med Kommentarer/Lindell/Haglind, 5997 , Lag $1994: 1117$ (kürfrig Svensk Kamov).

${ }_{1} 3$ Zu den Vorarbeiten näher Statens Offentliga Utredningar (SOU) 1993: 98 Partnerskap, 1993/94: LU 28. 
den erließ darüber hinaus jedoch schon 1987 ein Reformgesetz über homosexuell Zusammenlebende (lag om homosexuella sambor) ${ }^{r+}$, das auf eine Gleichstellung der homosexuellen eheähnlichen mit der heterosexuellen nichtehelichen Lebensgemeinschaft abzielt. Letztere war im selben Jahr im Gesetz über das gemeinsame Heim der nicht in ehelicher Lebensgemeinschaft Zusammenlebenden (lag om sambors gemensamma hem - sambolag) $)^{15}$ geregelt worden ${ }^{16}$ (dazu unter IV.I). Auf Island datiert das Lög um staðfesta samvist (Gesetz Nr. 87) vom I2. Juni 1996, auf das im vorliegenden Kontext nicht näher eingegangen werden konnte.

\section{Die Rechtslage in Dänemark ${ }^{17}$}

Die Einführung des Rechtsinstituts der registrierten Partnerschaft hat in Dänemark ihren Ausgang genommen ${ }^{18}$. Dänemark war der erste Staat, der sich dazu entschloß, eine entsprechende Rechtsfigur in seine Rechtsordnung aufzunehmen - zu einem Zeitpunkt, als die Einführung eines solchen Rechtsinstituts noch nirgendwo sonst geplant war ${ }^{19}$. Das dänische Gesetz über die registrierte Partnerschaft ist nach $\$ 6 \mathrm{Pal}$ DK am I. Oktober 1989 in Kraft getreten.

Im Unterschied etwa zu Schweden hat Dänemark das Zusammenleben von Personen gleichen Geschlechts außerhalb der registrierten Partnerschaft nicht gesondert geregelt. Dies liegt darin begründet, daß die Rechtsbeziehungen heterosexueller Paare (sog. papierloser Lebensgemeinschaften) ${ }^{20}$ - außerhalb der Ehe - nach dänischem Rechr grundsätzlich (nit Ausnahme einiger weniger Einzelbestimmungen in verschiedenen Gesetzen) gleichermaßen gesetzlich nicht geregelt sind ${ }^{2 t}$.

\section{Die Intention des dänischen Gesetzgebers}

Die gesetzgeberische Intention des "lov om registreret partnerskab « ist es, den rechtlichen Status des Zusammenlebens zweier gleichgeschlechtlicher Partner jenem gleichzustellen, den die Rechtsordnung zwei Personen unterschiedlichen $\mathrm{Ge}$ -

14 Svensk Forfattningssamling (SI:S) $1987: 813$

1s SFS 1987: 232

16 Dazu naher Agell, Die schwedische Gesetzgebung uber nichteheliche Gemeinschaften, FamRZ 1990, 817; Heilmann, Die nichteheliche Lebensgemeinschaft, JA I990, 116 (119ff. - Schweden); Radau, Die Gesetzgebung zur nichtehelichen Lebensgemeinschaft in Schweden, MDR 1989, 703; Spindler, Akruelle Neuregelungen des Rechts der nichtehelichen Lebensgemeinschaft in Schwcden, FamRZ $1988,913$.

$17 \mathrm{Vgl}$. dazu auch Wacke, Die Registrierung homoscxueller Partnerschaften in Dänemark, FamRZ 1990, 347 .

is Vgl. zuletzt auch die rechtspolitische Entwicklung in Frankreich, wo gegenwartig dic Einführung eines neuartigen Partnerschaftsvertrags (pacte de solidarité civile [pacs]) für außereheliche Verbindungen - von unverheirateten Personen (unabhängig von Geschlecht, Verwandtschaftsverhalmis und Beweggrund für ein Zusammenleben ohnc Trauschein) sich im Gesetzgebungsverfahren befindet - dazu Hénard, Sag ja zum Pakt, DIF ZEIT NR. ${ }_{4} 6$ vom s. November 1998, S.74. Zudem Neue Zürcher Zeitung vom 9. Oktober 1998 und NJW r998, Heft 46, XIVII (Frankreich: Die Ehe in Zeiten der Postmoderne). Zur Rechrslage in den Niederlanden: Titel s A (Het geregistreerd partnerschap), die Art. 8oa bis e des Bürgerlichen Gesetzbuches (Burgerlijk Wetboek).

19 In diesem Sinne auch der (schwedische) staatliche Untersuchungsbericht SOU $1984: 63$, S. 96 und 99 sowie die dänische betænkning nr. 1 r27/2988, S. 128 - Stcllungnahme der Arbeitsgrupponmehrheit.

20 Papirlose samlivsforhold.

21 So die Gesctzesvorarbeiten, betinkning nr. $1127 / 1988$, S. 124 - Stellungnahme der Ausschußminderheit und S. 129. 
schlechts einräumt ${ }^{22}$. Unter »Gleichstellung « wird die Zusicherung derselben Rechte und die Auferlegung gleicher Pflichten, wie sie unter Ehepartnern gelten, verstanden. Nach $\rrbracket_{3}$ Abs. 2 Pal DK finden grundsätzlich alle Regelungen der dänischen Gesetzgebung (mithin auch solche außerhalb des Ehe- und Erbrechts) ${ }^{23}$, die die Ehe « oder die »Ehepartner betreffen, auf registrierte Partnerschaften bzw. auf registrierte Partner entsprechende Anwendung (Grundsatz einer umfassenden rechtlichen Gleichstellung).

\section{Voraussetzungen einer Registrierung}

Eine wirksame Registrierung setzt zunächst voraus, daß beide Partner gleichen Geschlechts sind (so $\$ 1 \mathrm{Pal} D K$ ). Ausweislich der Motive ist der Gesetzgeber aber davon ausgegangen, daß eine sexuelle Orientierung nicht erforderlich ist, was ansonsten eine ausdrückliche Legaldefinition der Gleichgeschlechtlichkeit im Pal erforderlich gemacht hätte ${ }^{24}$. Eine solche Definition war weder erwünscht noch glaubte man, darüber letztlich Einigkeit erzielen zu können"s.

Ein gemeinsamer Wohnsitz der Partner wird nach dem Pal DK gleichermaßen nicht zur Voraussetzung der Registrierung gemacht.

Aus dem Gesetzeswortlaut des $₫$ I Pal DK folgt, daß allein eine Zwei-PersonenPartnerschaft registrierbar ist - nicht jedoch bspw. ein größeres Kollektiv ${ }^{26}$ registriert werden kann.

Versuche von Partnern nichtehelicher Lebensgemeinschaften zwischen einem Mann und einer $\mathrm{Frau}^{27}$, ihre Lebensgemeinschaft auf der Grundlage des Pal DK registrieren zu lassen, sind von den Registerbehörden frühzeitig zurückgewiesen worden.

Aus dem Verweis in $\$ 2$ Abs. I Pal DK auf Kapitel $x^{28}$ sowie die $\$ \${ }_{2} 2$ und ${ }_{13}$ Abs. I und Abs. 2 S. $\mathrm{I}^{29}$ des Gesetzes Nr. 256 über die Eingehung und die Auflösung der Ehe (Ehegesetz - lov om ægteskabs indgåelse og opløsning) ${ }^{30}$ aus dem Jahre 1969 folgt, daß die registrierungswilligen Partner jene Voraussetzungen zu erfüllen haben, die auch im Zusammenhang mit der Eingehung einer Ehe vorliegen müssen. So gilt etwa auch für registrierte Partnerschaften das Zustimmungserfordernis der Eltern und des Staatsamts" im Falle einer Registrierung minderjähriger Partner bzw. das Verbot der

22 Kommission zur Untersuchung der Situation Homosexueller in der Gesellschaft (kommissionen til belysning af homoscksuclles situation i samfundet) - betænkning nr. 1127/1988 (homoseksuelles vilkár).

2) Problematisch konnen hingegen auf vertraglicher Basis beruhende Regelungen zugunsten eines Ehepartners sein - erwa in Pensionsordnungen. Im Rechtsausschuß des Folketing wurde dazu scitens der Minderheir die Auffassung vertreten, daß Versicherungs- oder Pensionsordnungen so behandelt werden sollten, daß registrierte Partner mit Ehepartnern gleichgestellt werden. Industric- und Justizministerium vertraten hingegen die Ansicht, daß die Frage einer diesbezuglichen Gleichstellung der allgemeinen Vertragsauslegung vorbehalten bleiben müsse - dazu Rosholm, Skarrildhus Rapporten 1989, S. 66

24 Anmerkung zum Gesetzentwurf, Folketingstidende 1988/89, Anhang A, Spalte 2943.

25 -Es ware unakzeptabel, wenn Personen, die sich an die Behörden mit den Ziel wenden, eine registrierte Partnerschaft einzugehen, ggf. intime Verhaltnissc darlegen müßtena - si) betankning nr. $1127 / 1988$, $\mathrm{S} .114 \mathrm{f}$.

26 So kann etwa eine gleichgeschlechtliche Mehr-Personen-Wohngemeinschaft nicht durch Registrierung das gesetzliche Erbrecht unter ihren Mitgliedern zur Entstehung gelangen lassen - Dansk Karnov/ Nielsen, Pal $\$ 1$ Anm.1.

27 Zur Rechtsstellung nichtehelicher Lebensgemeinschaften näber Vindelov/Lund-Andersen/Nielsen, Retsstillingen for ugifte samlevende, 1988.

28 Ehebedingungen (xgteskabsbetingelser).

29 Die $\$ \$_{1} 2$ und 13 regeln die Prufung der Ehebedingungen.

$30 \mathrm{Vgl}$, zum Ehegesetz näher Danielsen/Hornslet/Knudsen, Ægteskabsloven med kommentarer, 2. udg., 1984; Lund-Andersen/Munck/Nørgaard/Højgaard Pedersen/Vesterdorf, Familieret, 4. udg, 1996; Nielsen/Vorstrup Rasmussen/Taksøe-Jensen, Familieretten, 2. udg., 1997.

31 Das Staatsamt hat regelmaßig bei seinen Entscheidungen berücksichtigt, ob die Frau schwanger ist oder 
Registrierung von Verwandten in direkter auf- oder absteigender Linie oder von Geschwistern. Des weiteren gilt das Verbot der Bigamie (d. h. der Neuregistrierung eines Partners, obwohl seine Ehe oder eine bereits früher registrierte Partnerschaft noch nicht geschieden bzw. aufgelöst wurde) ${ }^{32}$.

Dic Möglichkeit einer staatlich anerkannten kirchlichen Eheschließung ${ }^{33}$ besteht für gleichgeschlechtliche Partnerschaften nicht ${ }^{34}$.

Die nähere Ausgestaltung des Registrierungsverfahrens ist in den $\$ \$ 35$ bis 40 der Verordnung Nr. 662 vom I. August r 995 über die Eingehung der Ehe (bekendtgørelse om ægteskabs indgåelse) geregelt ${ }^{3 s}$. Die Registrierung erfolgt nach $\$ 39$ Abs. I in Anwesenheit von mindestens zwei Zeugen. Nach $\$ 40$ i.V.m. $\$ 39$ Abs. 2 dieser Verordnung ist eine Partnerschaft dann wirksam registriert worden, wenn die Registrierung - bei gleichzeitiger Anwesenheit und Erklärung der Partner - bei der zuständigen staatlichen Behörde erfolgt ist. Eine Registrierung der Partnerschaft (nach dänischem Recht) im Ausland ist ausgeschlossen.

Besonderheiten bestehen, wenn einer der registrierungswilligen Partner Ausländer ist: Nach dem ausdrücklichen Wortlaut des $\$ 2$ Abs. 2 Pal DK kann eine Registrierung nur dann erfolgen, wenn beide oder mindestens einer der Partner seinen Wohnsitz in Dänemark hat und dänischer Staatsangehöriger ist. Das Gesetz fordert mithin - im Unterschied zu einer Eheschließung - eigentlich die feste Beziehung wenigstens einer der Partner zu Dänemark. Der Sinn der Regelung liegt darin begründet, daß der Gesetzgeber die Registrierung rein ausländischer Partnerschaften in Dänemark als unzweckmäßig angesehen hatte, da dieser Registrierung im Heimatland der ausländischen Partner (bis auf weiteres) keinerlei Bedeutung zukomme ${ }^{36}$. Gleichwohl hat der Oberste Gerichtshof Dänemarks (højesteret) in einem Urteil vom 20. August $1993^{37}$ entschieden, daß die Registrierung einer Partnerschaft zwischen zwei Ausländern Gültigkeit beanspruche. Das Gericht begründete seine Auffassung damit, daß die Wirksamkeitsgründe einer registrierten Partncrschaft abschließend in $\$ 40$ der vorgenannten Verordnung über die Eingehung der Ehe normiert seien. Da die Wohnsitz- bzw. die Staatsangehörigkeitsfrage in $\$ 40$ jedoch keinen Niederschlag gefunden habe, stellte das Gericht fest, daß die Partnerschaft zwischen den beiden Ausländern nach dänischem Recht gültig registriert worden $\operatorname{se}^{3{ }^{8}}$.

einer der künftigen Ehepartner ein Kind hat, das mit in die Ehe eingebracht wird. Diese Umstande sind im Zusammenhang mir der Registrierung gleichgeschlechtlicher Partncr nicht zu beachten, da ausweislich der gesetzgeberischen Intention eine Gleichstellung der registrierten Partnerschaft mit der Ehe in bezug auf Kinder nicht gewünscht war und ist - Nielsen/Vorstrup Rasmussen/Taksøe-Jensen (Fn. 9), Kapitel i $\$ .2$ m.w.N.

32 Die registrierte Partnerschaft kann bei Auslandsbezügen insoweit allerdings andere Konsequenzen haben: $\mathrm{Da}_{\mathrm{a}}$ das Ausland die Registricrung zumeist nicht anerkennen wird, kann einer neuen im Ausland eingegangenen Ehe dort regelmäßig nicht - wegen der in Dänemark vorausgegangenen Registrierung - der Einwand der Bigamie entgegengehalten werden. Svenné Schmidt (Fn. 9, S. 4I) sieht in einem solchen Falle allein die Móglichkeit, daß der andere Partner eine Auflösung der Parnerschaft nach Maßgabe des Eherechts in die Wege leitet.

33 Nach $f$ is Abs. I des Ehegeserzes kann in Dänemark eine Ehe entweder durch einc kirchliche oder cinc burgerliche (Zivil-)Trauung eingegangen werden. Eine kirchliche Trauung innerhalb der danischen Volkskirche (dazu nachstehende Fußnote 34 ) erfolgt nach $\$ 17$ Abs. 1 Fhegesec\% durch deren Pfarrer. Kirchliche Trauungen durch andere Glaubensgemeinschaften werden von jenen Geistlichen vorgenommen, die dazu ermächtigt wurden ( $\$ 17$ Abs. 2 Ehegesecz).

34 Die Dänische Volkskirche (den danske folkekirke - zum rechtlichen Status näher Ring/Olsen-Ring (Fn. 7), Rn. 17rff.) diskuticr gegenwärtig die Möglichkeit einer (nachtraglichen) bloßen Segnung der registrierten Partnerschaft - rapport fra et af biskopperne nedsat udvalg vedrørende kirkelige velsignelse af registreret partnerskab (Registeret partnerskab, samliv og velsignelse).

3s Vgl. zudem den Runderlaß Nr. ${ }_{34}$ vom 2 s. Seprember 1989 uber die Eingehung der Ehe (cirkulare om xgteskabs indgåelse).

36 Folketingstidende $1988 / 89$, Anhang A, Spalte 1877 und $1880 \mathrm{f}$.

37 Sag II $18 / 1993 \mathrm{M}_{1}$ og $\mathrm{M}_{2}$ mod Civilretsdirektoratet = Ugeskrift for retsvæsen (UfR) $1993,849 \mathrm{f}$.

38 A.A. noch die Vorinstanz - Østre Landsret (r6. afdeling), Urteil vom I 2. Januar r993. Dazu Danielsen, Lov \& Rer 1993 Nr. 5, 20. 
Ohne hier näher auf die Entscheidung eingehen zu können, überrascht es doch, daß der Oberste Gerichtshof der Verordnungsregelung (die im Gleichklang mit den Vorgaben des Eherechts steht) einen Vorrang vor der ausdrücklichen Gesetzesregelung in $\$ 2 \mathrm{Abs} .2 \mathrm{Pal} \mathrm{DK}$ beigemessen hat. Auf die Frage, welche der Regelungen die speziellere sei, wurde in der Begründung nicht näher eingegangen.

\section{Rechtswirkungen der Partnerschaft}

Die registrierte Partnerschaft entfaltet nach $\rrbracket_{3} \mathrm{Pal} \mathrm{DK}$ grundsätzlich dieselben Rechtswirkungen wie die Ehe. Daraus resultiert, daß als Folge der Registrierung zwischen den Partnern eine gegenseitige gesetzliche Unterhaltspflicht begründet wird. A uf die Partner findet das eheliche Güterrecht Anwendung (d. h. ihr Vermögen stellt grundsätzlich Ausgleichs-, mithin Gemeinschaftsgut dar, sofern es sich nicht ausnahmsweise um Vorbehaltsgut handelt) ${ }^{39}$. Die Partner sind - ebenso wie Ehegatten $4^{4}-$ gesetzliche Erben. Im übrigen gelten sowohl die steuer- als auch die sozialrechelichen Vorschriften zugunsten von Ehegatten auch für registrierte Partner.

Ausnahmen von der grundsätzlichen Gleichstellung mit der Ehe resultieren aus $\rrbracket_{4}$ Pal DK. Nach $\S_{4}$ Abs. I Pal DK finden die Regelungen im Adoptionsgesetz Nr. 279 aus dem Jahre 1972 (lov om adoption) betreffend Ehepartner auf registrierte Partnerschaften keine Anwendung. Dies bedeutet, daß registrierte Partner nicht gemeinsam adoptieren können. Insoweit stellt sich jedoch die Frage, ob möglicherweise jeder von ihnen allein adoptionsberechtigt ist. Obwohl das dänische Pal diese Frage nicht beantwortet, geht die h.A. davon aus, daß auch einer der Partner einer registrierten Partnerschaft nicht berechtigt ist, allein zu adoptieren ${ }^{41}$.

Im übrigen bestimmt $\rrbracket_{4}$ Abs. 2 Pal DK, daß $\$ r_{1} S .2$ und $\$ r_{4}$ Abs. 4 des Gesetzes Nr. 387 über die elterliche Sorge und das Umgangsrecht vom I 4. Juni I99s (lov om forældremyndighed og samvær) auf registrierte Partnerschaften keine Anwendung finden.

$\$ 4$ Abs. 3 Pal DK schließt auch Bestimmungen der dänischen Gesetzgebung von der Anwendung auf registrierte Partnerschaften aus, die besondere Regelungen hinsichtlich eines Ehepartners treffen, die jedoch auf das Geschlecht dieser Person abstellen. Der Anwendungsausschluß erfaßt etwa Vaterschaftsregelungen sowie die Bestimmung über die "Schlüsselgewalt « der Ehefrau (! $)^{42}$ nach $\$$ I I Abs. I S. 2 des Gesetzes Nr. 56 über die Rechtswirkungen der Ehe (lov om ægteskabets retsvirkninger) vom I 8. März 1925 .

$\$ 4$ Abs. ${ }_{4}$ Pal DK bestimmt des weiteren, daß Regelungen in internationalen Übereinkommen keine Anwendung auf die registrierte Partnerschaft finden, es sei denn, die Vertragspartner der Übereinkommen haben eine entsprechende Weiterung gebilligt. Die Bestimmung des $\S_{4}$ Abs. 4 Pal DK wird jedoch entgegen ihrem ein-

39 Zum ehelichen Vermogensrecht in den nordischen Staaten naher Ring/Olsen-Ring (Fn. 7), Rn. $432 \mathrm{ff}$. 40 Das gesetzliche Erbrecht der Ehegatten ist im zwciten Kapite: ( $\$ 6$ und 7) des Erbgesetzes (arvelov Nr. 2 15) vom 3 r. Mai 1963 geregelt. Zum danischen Erbrecht naher Danielsen/Gaarden/Rogvi, Arveloven med kommentarer, 3. udg. 1991; Svenné/:Vargaard/Vesterdorf, Arveret, 2. udg., 1991; Taksøe-Jensen, Arveretten, 4. udg., 1994

41 Vgl. dazu Rosholm, Skarrildhus rapporten, 1989 , s.68 unter Bezugnahme auf die auch vom Zivilrechtsdircktorat (civilretsdirektorat) vertretene Auffassung. Ebenso Nielsen/Vorstrup Rasmussen/Taksøe-Jensen (Fn. 9), die jedoch unter Bczugnahme auf das Adoptionsgesctz argumenticren, daß auch ein einzelnes Ehepartner nicht allein adopticren konne; a.A. Sylvest Niclsen, R \& R 1991/8,60 (die den Schluß, ein registrierter Parner könne nicht allein adoptieren, fur zweifelhaft hält).

42 Sic! Eine Regelung, die entgegen des liberalen Zuschnitts des danischen Eherecht immer noch Bestand hat. 
deutigen Wortlaut, der keine Einschränkung im Hinblick auf das bloße Außenverhältnis Dänemarks zu seinen Vertragspartnern enthält, restriktiv ausgelegt ${ }^{43}$ : Regelungen in internationalen Verträgen, die einem Ehepaar Rechte zubilligen bzw. Verpflichtungen auferlegen, haben die dänischen Behörden im Innenverhältnis dänischer Staat zu registrierter Partnerschaft uneingeschränkt auch auf letztere anzuwenden, sofern diese Vorschriften registrierte Partnerschaften im Vergleich zu Ehepaaren weder bevorzugen noch diskriminieren. Diese Auslegung erfolgt vor dem Hintergrund, daß im Verhältnis der Konventionsstaaten zu Dänemark erstere vielfach einer in Dänemark registrierten Partnerschaft (wegen Verstoßes gegen den eigenen ordre public) die Anerkennung versagen werden.

\section{Die Auflösung der registrierten Partnerschaft}

Die Auflösung einer registrierten Partnerschaft ist in $\$ 5$ Pal DK geregelt. Danach gelten die Bestimmungen der Kapitel 3, 4 und 5 des Gesetzes über die Eingehung und die Auflöung der Ehe (Ehegesetz - dazu bereits unter II.2.) über die Anfechtung, die Trennung und die Scheidung (einschließlich der daraus resultierenden Vermögensverhältnisse, etwa die Leistung von Unterhalt) sowie das Kapitel 42 des Prozeßgesetzes (Rechtspflegegesetz Nr. I9 vom rr. April 1916 - retsplejeloven) grundsätzlich entsprechend ${ }^{44}$.

Keine Anwendung findet hingegen nach $\ 5$ Abs. 2 Pal DK die Regelung des $\ 43$ Abs. I Ehegesetz. Nach dieser Vorschrift haben (als Ausfluß des dänischen Staatskirchensystems ${ }^{45}$ ) die Ehepartner ein Recht darauf ${ }^{46}$, daß im Falle einer zerütteten Ehe ein Pfarrer schlichtet ${ }^{47}$. Unanwendbar ist zudem $\$ 46$ Ehegesetz, eine Vorschrift, die die Frage der elterlichen Sorge bei Trennung oder Scheidung der Ehepartner regelt. Der Ausschluß dieser Vorschrift ist Korrelar der Vorgabe in $\$ 4$ Abs. 2 Pal DK (oben II.3.), daß registrierte Partner kein gemeinsames Sorgerecht über Kinder haben können. Entgegen der Vorschrift von $₫ 448 \mathrm{c}$ des Prozeßgesetzes bestimmt $\$ 5$ Abs. 3 Pal DK, daß eine registrierte Partnerschaft - unabhängig vom Wohnsitz und der Staatsangehörigkeit der Partner - in Dänemark jederzeit aufgelöst werden kann ${ }^{48}$. Die jederzeitige Möglichkeit der Auflösung einer Partnerschaft in Dänemark liegt darin begründet, daß der Gesetzgeber davon ausgegangen ist, eine registrierte Partnerschaft würde regelmäßig im Ausland nicht aufgelöst werden können ${ }^{49}$.

43 Dansk Karnov/Nielsen, $\$_{4}$ Pal Anrn. 9.

44 Detailbestimmungen finden sich in der Verordnung Nr. 627 vorn 25 . September 1989 uber die Auflosung der Ehe (bckendtgørelsc om xgteskabs oplesning) und im Runderlaß Nr. ${ }_{3} 35$ vom 25 . September 1989 (cirkulare om rgteskabs oplesning).

45 Dazu naher Dübeck, Staat und Kirche in Dänemark, in: Robbers, Stast und Kirche in der Europäischen Union, 1995, S. $39 \mathrm{ff}$.

46 Vor dem Jahre i 989 war vor ciner Scheidung cine entsprechende Schlichtung sogar obligatorisch.

47 Dies schließt es jedoch nicht aus, daß ein Geistlicher - außerhalb der geseculichen Regelung - in W/ahrnehmung scines Seclsorgeauftrags eine entsprechende Schlichtung vornimmt, wenn er selbst dazu bercit

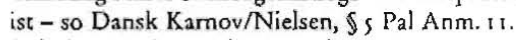

$48 \mathrm{Vgl}$. dazu auch $\$ 21$ der Verordnung Nr. 627 vom 29. September 1989.

49 Betærnkning nr. $1127 / 1988$, S. 120 
Norwegen war der erste Staat nach Dänemark ${ }^{\text {so }}$, der das Institut einer registrierten Lebenspartnerschaft anerkannt hat. Der Gesetzgeber entschloß sich aus Praktikabilitätserwägungen zu einer grundsätzlichen Gleichstellung der registrierten Partnerschaft mit der Ehe, da ansonsten eine Viclzahl von Regelungen - die gleichermaßen für homophile Paare in ökonomischer wie juristischer Hinsicht relevant sind - auf letztere keine Anwendung fänden, was die Auswahl des für registrierte Partner geltenden Rechts erschwert hätte"'. Das norwegische Lov om registrert partnerskap Nr. 40 vom 30. April 1993 ( $\mathrm{Pal} \mathrm{N}$ ) lehnt sich im wesentlichen an die vier Jahre zuvor in Dänemark erlassene Regelung an ${ }^{52}$.

\section{Das Homophilitätserfordernis}

Im Unterschied zu $₫ \times$ Pal DK bestimmt die Parallelvorschrift des $₫ \times$ Pal N, daß zwei "homophile» Personen desselben Geschlechts ihre Partnerschaft nach Maßgabe des $\mathrm{Pal} N$ registrieren lassen können. Der Hinweis auf eine Homophilität war vom Gesetzgeber ausdrücklich gewünscht worden, der mit dem Gesetz auf einen Schutz dieser Paare abzielte. Der ausdrückliche Hinweis auf die Homophilie führt in rechtlicher Hinsicht jedoch - vor allem auch im Rahmen der Registrierungspraxis - nicht zu einer Überprüfung der sexuellen Orientierung im Einzelfalls3.

\section{Voraussetzungen einer Registrierung}

Nach $\ 2$ Abs. r S. r Pal N gilt Kapitel I des Ehegesetzes (lov om ekteskap - Nr. 47) vom 4. Juli r $99 \mathrm{I}^{54}$ über die Voraussetzungen der Eingehung einer Ehe (vilkår for å inngå ekteskap) entsprechend (Volljährigkeit, Verbot der Bigamie und Partnerschaft zwischen nahen Verwandten). Voraussetzung für die Eingehung einer registrierten Partnerschaft ist es (wie auch bei der Ehe) nicht, daß die Partner zusammen wohnen bzw. ein sexuelles Verhältnis miteinander haben.

In Anlehnung an $\$ 2$ Abs. 2 Pal DK bestimmt $₫ 2$ Abs. 3 Pal N, daß eine Registrierung der Partnerschaft nur erfolgen kann, wenn einer oder beide Partner ihren Wohnsitz in Norwegen haben und mindestens einer der beiden norwegischer Staatsbürger ist.

so Der Entwurf eines entsprechenden norwegischen Gesetzes datiert allerdings bereits schon aus dem Jahre 1989/90 - Dokument ar. 8:50 (1989/90 - Forslag fra stortingsrepresentantene ... om lov om registrert partnerskap). Dieser Parlamentsentwurf war bereits im Großen und Ganzen identisch mit dem später verabschiedeten Gesetz.

51 In diesem Sinne Ot.prp. nr. 32 (1992/1993), s. 16

s2 Urspringlich waren insgesant vier unterschicdliche Modelle Gegenstand der gesetzgeberischen Diskussion: (1) Eine gesetzliche Gleichstellung der Partnerschaft mit der Ehe. (2) Eine Regelung, die lediglich gewisse okonomische Verhältnisse berúcksichtigen sollte. (3) Eine Erwciterung des Gesetzes über den gemeinsamen Haushalt - Lov om rett til felles bolig og innbo når husstandsfellesskap opphører (Gesetz Nr. 4s vom 4. Juli 1991). Dieses Gesetz finder nach seinem \$ I dann Anwendung, wenn zwei oder mehrere unverheiratete Personen, dic alter als 18 Jahre sind, in einem Haushalt mindestens zwei Jahre zusammengewohnt haben. Sind die genannten Voraussetzungen erfuilth kann im Falle des Todes (einer der Personen) bzw. der Auflösung des gemeinsamen Haushaltes nach den $\$ 2$ und 3 der andere Partner in das Wohnreche (Mierverhältnis) eintreten und ggf. das Wohnmobiliar ubernehmen. (4) Ein gencinsame's Gesetz uber das Zusammenwohnen hetero- bzw. homophiler Paare. Zu diesen Modellen nä?ter Ot.prp. nr. 32 (1992/1993), s. 13 ff. und Strandbakken, Lov om registrert partnerskap, JV s/6 1993, 300 (3 I 4 ff.).

53 So ausdrücklich Norsk Karnov/Strøm Bull, $\$$ I Pa! N Anm. 1 .

s4 Zum norwegischen Farnilienrecht Ring/Olsen-Ring (Fn. 7), Rn. 408. 
Die Vorgaben des Ehegesetzes über die Prüfung der Ehevoraussetzungen sowie die Regeln über die Eingehung der Ehe (Trauung) finden auf die Partnerschaft keine Anwendung. Stattdessen wurden auf der Grundlage von $\rrbracket_{2} \mathrm{Abs} .4 \mathrm{Pal} N$ vom Kinder- und Familienministerium inhaltlich vergleichbare Regelungen für die Part-

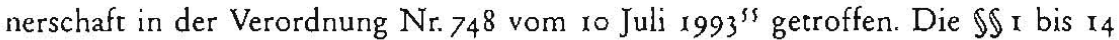
dieser Verordnung regeln im einzelnen die Prüfung der Registrierungsvoraussetzungen. Die Prüfung der Voraussetzungen und die Registrierung selbst können nur beim örtlich zuständigen "Notar" (notarius publicus) $)^{56}$ erfolgen $(\mathbb{}(\mathrm{s})$ ). Eine kirchliche und damit wegen des nordischen Staatskirchensystems grundsätzlich eigentlich statthafte - Trauung ist gegenwärtig (wegen innerkirchlicher Widerstände in Norwegen) nicht möglich. Im Falle einer Änderung der kirchlichen Einstellung wäre durch eine bloße Revision des $₫ 1$ is der Verordnung auch eine rechtswirksame "kirchliche Registrierung" möglich"s7.

Es war zunächst überlegt worden, dem "Notar « ein Recht auf Verweigerung der Registrierung aus persönlichen Gewissensgründen einzuräumen. Der Gesetzgeber hat letztlich davon abgesehen, da die Registrierung einer Partnerschaft eine verwaltungsmäßige Aufgabenstellung sei, bei der es keine Norwendigkeit für eine solche Weigerung geben könne ${ }^{58}$. Strandbakken ${ }^{99}$ weist im übrigen zutreffend darauf hin, daß ein Weigerungsrecht des Richters als Notarius publicus auf eine Zensurinstanz gegenüber dem Gesetzgeber hinauslaufen würde.

\section{Die Rechtsfolgen der Registrierung}

Die wesentliche Regelung des $\rrbracket_{3}$ Pal $N$ bestimmt, daß die Registrierung der Partnerschaft dieselben Rechtswirkungen wie die Eingehung einer Ehe entfaltet. Der Ausnahmebereich des $\rrbracket_{4} \mathrm{Pal} N$ ist noch enger als die Vergleichsregelung des $\rrbracket_{4} \mathrm{Pal} D K$ gefaßt: Auf die registrierte Partnerschaft finden nur die Vorschriften des Adoptionsgesetzes (lov om adopsjon - Nr. 8) vom 28. Februar 1986 über Ehepartner keine Anwendung ${ }^{60}$. Die Ausnahme wurde damit begründet, daß jede Diskussion über die Möglichkeit einer Adoption in den Bedürfnissen des Kindes ihren Ausgangspunkt nehmen müsse ${ }^{61}$.

Obgleich keine sonstigen Einschränkungen hinsichtlich der Gleichstellung mit der Ehe erfolgt sind, ist zu berücksichtigen, daß auch in Norwegen eine Reihe von Vorschriften, die auf Ehepartner Anwendung finden, an das Geschlecht anknüpfen. $\mathrm{Ob}$ solche Vorschriften auf die registrierte Partnerschaft uncingeschränkte Anwen-

is BFD I $993-07-10748$.

s6 Wegen der nicht ertolgten Rezeption des romischen Rechts in den nordischen Staaten ist dort kein dem deutschen Recht vergleichharer Notarberuf als selbstandige, unabhängige und ausschließliche Tätigkeit ublich - dazu näher Cornelius, Das dänische Notarwesen, DNotZ r996, 352 (354); Ring/Olsen-Ring (Fn, 7), Rn. 214 . Dic notarielle Tätigkeir wird ublicherweise durch den Richter wahrgenommen, der die eigentliche Aufgabenstellung auf einen Gerichtsassessor delegieren kasn. Im Rahmen der Ausübung notarieller Geschäfte wird der Richter dann aber nucht als Richter, sondern als Notar (notarius publicus) tatig.

S7 So Standbakken, Lov om registrert partnerskap, JV s/6 1993, 300 (329)

58 Gesetzesmaterialien - Ot.prp. nr. 32 (1992/93), s. 32 1. sp.

s9 Lov om registrert partnerskap, JV s/6 1993, 300 (331).

60 Die einzige Ausnahme von der grundsatzlichen Gleichstellung in $\S_{3}$ erfaßt nach $\S_{4} \mathrm{Pal}_{\mathrm{N}} \mathrm{N}$ die Adoptionsberechtigung der Partner mit der Folge, daß diese weder zusammen ein (fremdes) Kind noch ein Partner allein das Kind des anderen Partners adoptieren kann.

6. So Ot.prp. nr. 32 (1992/93), s. 33 . 
dung finden, bedarf hier - im Unterschicd zu Dänemark, wo $\rrbracket_{4}$ Abs. 3 Pal DK eine

Anwendung ausdrücklich ausschließt - einer Beurteilung im konkreten Einzelfall. In diesem Zusammenhang weist etwa Strøm Bull ${ }^{62}$ - jedoch ohne nähere Begründung darauf hin, daß zwei in einer Partnerschaft registrierten Partnern nicht das Recht auf eine künstliche Befruchtung nach Maßgabe des entsprechenden Gesetzes (Lov om kunstig befruktning - Nr. 68) vom r2. Juni 1987 zusteht.

\section{Die Auflösung der Partnerschaft}

Aus $\rrbracket_{3}$ Abs. 2 Pal N folgt, daß die registrierte Partnerschaft in Übereinstimmung mit den Regeln in Kapitel 4 des Ehegesetzes aufgelöst werden kann. Dies hat etwa zur Folge, daß die Auflösung einer Partnerschaft nach einem Trennungsjahr erfolgen kann. Ebenso wie nach $\llbracket s$ Abs. 3 Pal DK kann gemäß $₫ s$ Pal N eine registrierte Partnerschaft, die in Norwegen eingegangen wurde, jederzeit und unabhängig von $\$ 4$ I 9 a des Zivilprozeßgesetzes vor einem norwegischen Gericht auch wieder aufgelöst werden.

\section{Die Rechtslage in Schweden}

Die Rechtslage in Schweden unterscheidet sich von jener in Dänemark und in Norwegen in zweierlei Hinsicht: Zum einen dadurch, daß bereits vor dem Erlaß eines Partncrschaftsgesetzes (unter 2.) ein solches über homosexuell Zusammenlebende (I.) verabschiedet worden war, zum anderen aber auch dadurch, daß die Vorschriften des Lag ( 1994 : I I 7 ) om registrerat partnerskap (PaLS) weit umfänglicher ausgefallen sind ( 7 Paragraphen in drei Kapiteln) als in den dänischen bzw. den norwegischen Parallelregelungen. Daneben existiert eine gesonderte Verordnung über die Registrierung von Partnerschaften (förordning [1994 : 134I] om registrering av partnerskap).

\section{Das Gesetz über homosexuell Zusammenlebende ${ }^{63}$}

Das schwedische Reformgesetz über homosexuell Zusammenlebende ${ }^{64}$, das bereits sieben Jahre vor dem Pal S in Kraft getreten ist, zielt auf eine rechtliche Gleichstellung der homosexuellen mit der heterosexuellen nichtehelichen Lebensgemeinschaft ${ }^{65}$. Dieses Gleichstellungsgesetz trat gleichzeitig am x. Januar 1988 mit dem Gesetz über das gemeinsame Heim der nicht in ehelicher Lebensgemeinschaft Zusammenlebenden $^{66}$ in Kraft. Die gesetzgeberische Intention besteht darin, das homosexuelle

62 Norsk Karnov/Strom Bull, $\$_{4}$ Pal N Anm. 7 unter Bezugnahme auf die Gesetzesvorarbeiten in Ot.prp. nr. $32(1992 / 93)$ s. $35 \mathrm{f}$.

63 Dazu nahcr Widegren/Ytterberg, Homosexuelia sambor - innebörd och mottagande av en ny rattsfigur, SvJT 1990, 491; Agell, Äktenskap, samboendc, partnerskap, a.a.O.; Svensk Karnov/Brattgård, Lag (1987:813) om homosexuclla sambor; Tottic, Aktensikapsbalken och promulgationslag, 1990, S. $643 \mathrm{ff}$. 64 Iag $1987: 813$ om homosexuella sambor.

6) Die Vorarbeiten 7u diesem Gesetz finden sich im Bericht $*$ Homosexuelle und Geselischaft" (homosexuella och samhällat), SOU $1984: 63$, sowie in prop. 1986/87:124.

66 Lag 1987:232 om sambors gemensamma hem (sambolag) - dazu Ring/Olsen-Ring (Fn. 7), Rn. $456 \mathrm{ff}$. 
Zusammenleben ideologisch voll zu akzeptieren. Darüber hinaus beruht es auf der praktischen Ûberlegung, daß die Partner in zahlreichen homosexuellen Verhältnissen einen gemeinsamen Haushalt haben, ein gemeinsames Zuhause aufbauen und die wirtschaftlichen Verhältnisse der Partner in derselben Weise wie bei heterosexuell Zusammenlebenden verflochten werden ${ }^{67}$. Das Gesetz über homosexuell Zusammenlebende trifft keine materiellen Einzelregelungen. Vielmehr verweist das Gesetz auf eine Vielzahl von Gesetzen und Einzelvorschriften, die auf homosexuelle Lebensgemeinschaften entsprechende Anwendung finden. Über dieses Verweisgesetz finden insbesondere die Regelungen des Gesetzes über das gemeinsame Heim der nicht in ehelicher Lebensgemeinschaft Zusammenlebenden auch auf homosexuell Zusammenlebende Anwendung.

\section{Die registrierte Partnerschaft des schwedischen Rechts ${ }^{68}$}

\section{a. Die Registrierung der Partnerschaft}

Die Registrierung der Partnerschaft ist im ersten Kapitel Pal S sowie - mit Detailbestimmungen - in der Verordnung über die Registrierung von Partnerschaften ${ }^{69}$ geregelt.

I: Pal S bestimmt, daß zwei Personen desselben Geschlechts ihre Partnerschaft registrieren lassen können und entspricht damit der Regelung des $₫$ r Pal DK. Die Vorschrift verlangt weder eine gemeinsame Haushaltsführung noch geschlechrliche Beziehungen oder eine bestimmte sexuelle Orientierung ${ }^{70}$. Das Staatsangehörigkeitsund Wohnsitzerfordernis nach $\mathrm{r}: 2 \mathrm{Pal} \mathrm{S}^{\prime \prime}$ ist wie im dänischen ( $\mathbb{2}$ Abs. 2 Pal DK) bzw. norwegischem Recht ( $\$ 2$ Abs. 3 Pal $N$ ) geregelt. Im Unterschied zu den Parallelregelungen in Dänemark und Norwegen erfolgt im schwedischen Partnerschaftsgesetz jedoch in gesetzestechnischer Hinsicht kein Verweis auf die Bestimmungen des Ehegesetzes ${ }^{72}$. Vielmehr wird dessen Inhalt im Pal S wiederholt und teilweise modifiziert. Die Registrierung erfolgt gemäß I:6 Pal S in Anwesenheit von Zeugen bei gleichzeitiger Anwesenheit der zu registrierenden Partner ( $1: 7$ Abs. I Pal S): Nachdem jeder der beiden Partner in die Registrierung eingewilligt hat, erklärt der zuständige Registerbeamte ${ }^{73}$ sie zu registrierten Partnern ${ }^{74}$.

67 Agell, Fn. 16, Kapitcl 14.3 unter Bezugnahme auf die Gesetzesvorarbeiten in prop. 1986/87:124, S. 43. 68 Zur Vorgeschichte des schwedischen Parnerschaftsgesetzes naher Agell, Áktenskap, Samboende, Partncrskap, 2. udg., 1999, Kapitel 14.1 .

69 Forordning 1994:1 341 om registrering av partnerskap.

70 In diesem Sinne Svensk Kamov/Lindell/Haglind, s:s Pal S Anm. $\mathrm{r}$.

71 Eine Berufung auf die schwedische Staatsbürgerschaft (zur Staatsbürgerschaft näher Ring/Olsen-Ring (Fn. 7), Rn. s1 7 ff.) ist naturlich auch Personen mit doppelter Staatsangehörigkeit moglich - so klarsteliend Svensk Karnov/Lindell/Haglind, r:2 Pal S Anm. 2.

72 Zurn schwedischen Fhegeserz naher Ring/O!sen-Ring (Fn. 7), Rn. $410 \mathrm{ff}$.

73 Zuständiger Registcrheamter ist nach r:8 Pal S entweder ein Richter des erstinstanzlichen Gerichts (tingsrätt .. zum schwedischen Gerichtsaufbau Ring/Olsen-Ring (Fn. 7), Rn. 141 Ht.) oder cine Person, die vom Provinzia!rat (lansstyrelse - als oberstes staatliches Verwaltungsorgan einer der ${ }_{4}$ Provinzen Schwedens fungicrt der Provinzialrat mit einem Landeshaupumann [landshovding] an der Spitze [dazu Ring/Olsen-Ring (Fn. 7), Rn. 126]) dazu bestimmt wurde.

74 Für das Registrierungsverfahren gelten im einzelnen nach 1:9 Abs. 1 Pal S die Bestimmungen des 4:5, 7 und 8 AktB. Dies hat zur Folge, daß der Regıstrierungsbeamte sich zu vergerwissern hat, daß cine Prüfung von Registrierungshindernissen - mit negativem Befund - vier Monate vor der Registrienng erfolgt ist. Die Partner erhalter nach der Registrierung eine Registrierungsurkunde. Zudem hat eine Benachrichtigung der Steuerbebörde - die die Vorprufung vorgenommen hat - über die durchgefuhrte Registrierung zu erfolgen. Geriaß 1:9 Abs. 2 Pal S kann ein Buschluß des Registerbeamten im Klagewege (rach 1 5:3 und 4 ÄktB) angefochten werden - dazu naher Svensk Karnov/Lindell/Haglind, i:9 Pal Arm. 12. 
Im Hinblick auf die international-privatrechtliche Seite ciner Registrierung verweist r:9 Abs. 3 PaL S auf r:4 des Gesetzes über gewisse internationale Rechtsverhältnisse betreffend die Ehe und Vormundschaft ${ }^{25}$. Dies hat folgende Konsequenzen: Die Registrierung vor einer schwedischen Behörde hat nach schwedischem Recht zu erfolgen. Eine im Ausland registrierte Partnerschaft kann beim Vorliegen bestimmter Voraussetzungen in Schweden anerkannt werden. Die schwedische Regierung kann die Voraussetzungen dafür schaffen, daß Registrierungen nach schwedischem Recht im Ausland erfolgen können und gleichermaßen in Schweden nach ausländischem Recht. Die Registrierungsverordnung bestimmt in $\$ 4$ die nach $\$ s$ zu protokollierende Registrierungsformel des Registerbeamten.

\section{b. Die Auflösung der Partnerschaft}

Die registrierte Partnerschaft endet nach 2: I PaL S mit dem Tod eines der Partner oder durch die Entscheidung eines Gerichts. Insoweit finden gemäß 2:2 PaL S die Vorschriften des fünften Kapitels des Ehegesetzes über die Ehescheidung (äktenskapsskillnad) entsprechende Anwendung ${ }^{76}$. Ein entsprechendes Partnerschaftsverfahren muß stets vor einem schwedischen Gericht geführt werden, falls die Registrierung nach Maßgabe des PaL S erfolgt ist (so 2:4 PaL S). Diese Vorgabe soll wie die Parallelregelungen des dänischen und norwegischen Rechts gewährleisten, daß eine nach schwedischem Recht registrierte Partnerschaft auch aufgelöst werden $\mathrm{kann}^{77}$.

\section{c. Die Rechtswirkungen der Partnerschaft}

Die Rechtswirkungen einer registrierten Partnerschaft sind im einzelnen im dritten Kapitel PaL S geregelt. Vergleichbar der Rechtslage in den anderen hier untersuchten skandinavischen Staaten bestimmt 3:I PaL S, daß eine registrierte Partnerschaft grundsätzlich dieselben rechtlichen Wirkungen wie eine Ehe entfaltet. Sofern gesetzliche Bestimmungen auf eine Ehe bzw. auf Ehepartner Bezug nehmen, gelten diese regelmäßig gleichermaßen für die Partnerschaft bzw. für die registrierten Partner. Ausnahmen von der grundsätzlichen Gleichstellung der Partnerschaft mit der Ehe sind in $3: 2$ bis 4 PaL $S$ geregelt.

3:2 Abs. I S. I PaL S bestimmt, daß registrierte Partner weder gemeinsam noch jeder für sich nach dem vierten Kapitel des Elterngesetzes (föräldrabalk) ${ }^{8}$ ein Kind adoptieren können ${ }^{79}$. Registrierte Partner können auch nicht dazu ausersehen werden, gemeinsam die elterliche Sorge über einen Minderjährigen nach 6:10a des Elterngesetzes auszuüben (3:2 Abs. i S. 2 PaL S). Die zuletzt genannte Vorschrift schließt jegliche, auf eherechtlicher Grundlage basierende Regelung eines gemeinsamen Sor-

\footnotetext{
75 Lag om vissa internationale rattsforhảllanden rörande aktenskap och formynderskap (1904:26).

76 So finden etwa auch die Regelungen über einc Bedenkzeir (betänketid [ $5: 2 \mathrm{ff}$. $\ddot{\mathrm{k}} \mathrm{ktB}]$ - dazu naher Ring/ Olsen-Ring (Fn. 7), Rn. ${ }_{424}$ f.) entsprechende Anwendung.

77 So Svensk Karnov/Lindell/Haglind, 2:4 PaL S Anm. 17.

78 Vom 20. Juni 1949, in Kraft getreten am I. Januar : 950 (so $\$ 1$ des Einfuhrungsgesetzes zum Elterngesetz1949:382).

79 Zur Adoption nach schwedischem Reche näher Ring/Olsen-Ring (Fn. 7 ), Rn. $462 \mathrm{f}$. und $467 \mathrm{ff}$
} 
gerechts für registrierte Partner aus ${ }^{80}$. Nach 3:2 Abs. 2 PaL S finden auch die Gesetze über Insemination ${ }^{81}$ sowie über Befruchtung außerhalb des Körper ${ }^{82}$ auf registrierte Partner keine Anwendung. Das Adoptions- und Inseminationsverbot für registrierte Partner wird meist damit begründet, daß ein Kind ein Bedürfnis sowohl nach einer Mutter als auch nach einem Vater habe. Unter Berücksichtigung des Kindeswohls sollte es also nicht $z$ wei Elternteile desselben Geschlechts haben ${ }^{6_{3}}$.

Vorschriften des Eherechts, die für Ehepartner gelten und deren Anwendung eine Sonderbehandlung des einen Ehegatten nur aufgrund seines Geschlechts vorsehen, finden auf registrierte Partner keine Anwendung (so 3:3 $\mathrm{PaL} \mathrm{S})^{84}$.

\section{International-privatrechtliche Konsequenzen}

Die in Skaninavien seit einem Jahrzehnt gesammelten Erfahrungen mit dem Rechtsinstitut der registrierten (Lebens-) Partnerschaft machen vor allem eines deutlich: Die skandinavischen Gesetzgeber waren und sind sich seit Anerkennung der registrierten Partnerschaft stets ihrer rechtlichen "Insellage « bewußt gewesen.

Im grenzüberschreitenden Rechtsverkehr wird die Einführung eines neuen Rechtsinstituts zwangsläufigerweise Komplikationen nach sich ziehen. Bogdan ${ }^{8 s}$ weist jedoch zutreffend darauf hin, daß allein dieser Umstand nicht dazu führen dürfe, von der Neuschaffung rechtspolitisch gewünschter Rechtsinstirute Abstand zu nehmen.

Die weitaus meisten Staaten werden auch weiterhin daran festhalten, neben der bürgerlichen Ehe kein vergleichbares Rechtsinstitut für gleichgeschlechtliche Paare anzuerkennen. Auch künftig dürfte dieses Institut in der internationalen Rechtsgemeinschaft vielfach als Verstoß gegen den ordre public (der nationalen Rechtsordnung) verstanden werden, unabhängig davon, welchen Rechtskreis man jeweils betrachtet. Selbst für den Fall, daß eine Rechtsordnung dem skandinavischen Modell grundsätzlich nicht ablehnend gegenüber steht, werden vielfach einschlägige Kolljsions- und Anerkennungsregeln fehlen ${ }^{86}$. Ein weiteres Problem kann daraus resultieren, daß das Institut - bei grundsätzlicher Anerkennung - von Rechtsordnung zu Rechtsordnung ggf. mit einem unterschiedlichen Inhalt belegt wird.

Der deutsche Gesetzgeber muß sich daher darüber klar sein, daß nach Inkrafttreten eines entsprechenden Gesetzes auch Deutschland - zusammen mit einigen wenigen anderen Staaten - im internationalen Kontext eine Außenseiterrolle spielen wird.

Bei jedem Auslandsbezug mit Staaten, die das Rechtsinstitut nicht anerkennen, wird der familien- und erbrechtliche Status in Deutschland registrierter Partner wahrscheinlich aucb weiterhin keine rechtliche Anerkennung erfahren. Selbst im interskandinavischen Kontext, d. h. der Staaten, die für ihre Rechtsordnung registrierte

80 So Svensk Karnov/Lindell//Haglind, 3:2 PaL S Anm. 19.

8x Lag 1984:1 140 om insemination - dazu Ring/Olsen-Ring (Fn. 7), Rn. 476

82 Lag 1988:711 om befruktning utanför kroppen - dazu Ring/Olsen-Ring (Fn. 7), Rn. 477.

83 Dazu naher $\Lambda_{\text {gell }}$ (Fn. 16), Kapitel 14.2 unter Bezugnahme auf den Bericht des Gesetzgebungsausschusses in LU $1993 / 94: 28$, S. 23 ff.

84 Diesc Vorschrift nimmt Bezug auf eherechtliche Regelungen, die trotz der Liberalisierungstendenzen des Familienrechts immer noch verheirateten Frauen oder Witwen Sonderrechte wie erwa ökonomische Vorteile einräumen - Svensk Karnov/I.indell/Haglind, 3:3 PaL S Anm. 20.

8s Registrerat partnerskap och svensk internationell privatratc, SvJT 1994, 773 (783).

86 In diesem Sinne Bogdan, Registrerat partnerskap och svensk internationell privatratt, SvJT 1994, 773 (774). 
Partnerschaften anerkennen, bestehen international-privatrechtliche Unsicherhei$\operatorname{ten}^{87}$.

Im Verhältnis zu den Staaten, die das Rechtsinstitut anerkennen, bleibt zu überlegen, ob eine nicht formale de facto Anerkennung im Gegenseitigkeitsverhältnis ausreichend ist oder aber im Interesse der Rechtssicherheit eine bilaterale bzw. multilaterale Anerkennung der registrierten Partnerschaft im Konventionswege anzustreben ist.

Vor diesem Hintergrund gebietet es die Fürsorgepflicht des deutschen Gesetzgebers, der ein solches Gesetz initiiert, die registrierungswilligen Partner nicht in einer scheinbaren Sicherheit zu wiegen. Diese sollten über die Konsequenzen einer Registrierung im Falle eines Auslandsbezugs aufgeklärt werden. Es bleibt den Partnern dann unbenommen, soweit möglich, sich auf privatrechtlicher Grundlage (etwa im Wege von Testamenten, Versicherungen und anderen Individualabreden) abzusichern.

Michael W. Schröter

Mensch, Erde, Recht

\author{
Grundfragen ökologischer Rechtstheorie
}

Die ökologische Krise hat innerhalb der Rechtswissenschaft eine breite Diskussion um eine angemessene Reaktionsweise ausgelöst. Die hierbei entwickelten Konzepte unterscheiden sich jedoch beträchtlich. Insbesondere ist die Frage umstritten, ob Wertesysteme allein auf den Menschen bezogen sein müssen (Anthropozentrik) oder darüber hinausgehen können (Ökozentrik).

Der Verfasser gibt zunächst einen umfassenden Überblick verschiedener Konzepte ökologischer Rechtstheoric und entwirft anschließend einen eigenen Ansatz, der von seinen philosophischen Grundlagen bis zu den praktischen Konsequenzen ausgearbeitet wird.

Die Möglichkeit ökozentrischen Denkens wird in den Mittelpunkt gestellt. Im Ergebnis steht der Entwurf einer ökologischen Diskursethik. Abschließend werden die praktischen Umsetzungsmöglichkeiten ökozentrischer Rechtsvorstellungen diskutiert und Normierungsvorschläge unterbreitet.

Das verständlich geschriebene Werk, das Erkenntnisse aus Rechtswissenschaft, Politik und Philosophie einbezieht, richtet sich an alle Interessierten, auch außerhalb der juristischen Fachkreise. Der Verfasser hat bereits mehrere Publikationen zu Fragen der ökologischen Ethik und des Umweltrechts veröffentlicht.

1999, 283 S., brosch., 79,- DM, 577,- oS, 72,-sFr, ISBN 3-7890-5952-8

(Studien zur Reehtsphilosuphie und Rechtstheorie, Bd. 21)

\title{
NOMOS Verlagsgesellschaft
} 76520 Baden-Baden

87 Dazu naher Strandbakken, Lov om registrert partnerskap, JV s/6 1993, 300 ( 344 ff. - zum norwegischen Recht); Bogdan, Registrerat partnerskap och svensk incernationell privatratt, SvJT 1994, 773 (zum schwedischen Recht). 